\title{
KRAUSISMO, LAICIDAD E INNOVACIÓN EDUCATIVA: PROPUESTAS PEDAGÓGICAS \\ EN EL RÍO DE LA PLATA (1889-1906). \\ UN ESTUDIO BIBLIOGRÁFICO
}

SUSANA MONREAL

Universidad Católica del Uruguay

La influencia del krausismo en el pensamiento jurídico y pedagógico de varios países europeos es innegable, cuanto más su formidable influjo en la España de la segunda mitad del siglo XIX. Lo mismo puede decirse de la influencia mediatizada y adaptada, pero cierta, de las ideas de Krause y sus discípulos en Iberoamérica, incluyendo a los países del Río de la Plata.

Sobre la incidencia del krausismo en Iberoamérica conviene realizar dos precisiones. Primeramente, el krausismo no se manifestó como una filosofía rigurosa y bien definida, sino como una matriz ideológica, como una corriente más del pensamiento espiritualista y liberal, que aglutinó, en la segunda mitad del siglo XIX, a diversas manifestaciones filosóficas.
Algunos estudiosos se refieren a ellas como «espiritualismo»o «romanticismo» ${ }^{1}$. En segundo lugar, de las diversas doctrinas filosóficas -también del krausismo- se tomó los aspectos que respondían a necesidades y cuestiones que las repúblicas en formación se planteaban. Por esta razón, la metafísica krausista tuvo un desarrollo limitado en la región, mientras que otros aspectos del sistema lograron muy amplia acogida. En resumen, debe reconocerse la influencia del krausismo, pero no sobrestimarla, sobre todo su presencia en «estado puro» ${ }^{2}$. Este trabajo se focaliza en el contexto de los avances de la secularización en el Río de la Plata; en las relaciones entre krausismo y laicidad, desde la perspectiva del krausoinstitucionismo -es decir,

\footnotetext{
${ }^{1}$ Arturo A. Roig, Los krausistas argentinos, Buenos Aires, El Andariego, 2006, p. 11; Antolín Sánchez Cuervo, Krausismo en México, México, UNAM-Facultad de Filosofía y Letras, 2004, p. 79; Leopoldo Zea, El positivismo en México. Nacimiento, apogeo y decadencia, México, Fondo de Cultura Económica, 1975, p. 315; Arturo Ardao, Batlle y Ordóñez y el positivismo filosófico, Montevideo, Ediciones Número, 1951, p. 156.

${ }^{2}$ Gabriela Ossenbach Sauter, «La presencia del krausismo en el Ecuador. Su papel en el proceso de secularización del Estado y la sociedad", en: T. Rodríguez de Lecea (coord.), El krausismo y su influencia en América Latina, Madrid, Fundación Friedrich Ebert-Instituto Fe y Secularidad, 1989, p. 252.
} 
el krausismo vinculado a la Institución Libre de Enseñanza-; y en la influencia de esta corriente en propuestas pedagógicas innovadoras que se plantearon en el Río de la Plata a fines de la década de 1880 y comienzos de los '90. Hemos realizado nuestro estudio a partir de trabajos de relevamiento y de análisis bibliográficos en bibliotecas y publicaciones pedagógicas de Buenos Aires y Montevideo.

\section{Krausismo y laicidad}

El krausismo se origina con la persona y las enseñanzas de Karl Christian Friedrich Krause, nacido a fines del s. XVIII en Sajonia ${ }^{3}$. Krause desarrolló una agitada vida académica y, si bien no logró ocupar un lugar de reconocimiento en la vida universitaria alemana, atrajo discípulos que difundieron, casi con devoción, su pensamiento. Las relaciones de amistad, las tareas intelectuales comunes y la complicidad filosófica alimentaron lo que Enrique M. Ureña ha denominado el «triángulo krausista germano-belga-es- pañol $»^{4}$. Sobre todo a través de su discípulo Enrique Ahrens, el pensamiento de Krause se instaló y se enseñó en Bruselas, en la Universidad Libre, fundada en 1834. Las obras en francés de Ahrens y de su discípulo belga Guillermo Tiberghien, así como las traducciones al español de las mismas, representaron «un vínculo esencial y vivo» entre el krausismo belga y los krausismos hispánicos, incluso ibéricos ${ }^{5}$.

Desde mediados del siglo XIX, el krausismo ingresó en Iberoamérica como una corriente renovadora del espiritualismo de inspiración cousiniana y como filosofía de combate, ante la difusión del positivismo. El krausismo resultaba atractivo por ser un sistema amplio y armónico, que respondía a las inquietudes de los hombres de su tiempo. También ganó adeptos por ser un sistema espiritualista, que permitía a los liberales desligarse del dogma católico y de los preceptos de la moral cristiana, adoptando un deísmo fundado en la razón y una estricta moral de inspiración racional, la moral del deber. Finalmente, la filosofía de Krause despertó adhesión

\footnotetext{
${ }^{3}$ Sobre Krause y su sistema filosófico: 0. Carlos Stoetzer, Karl Christian Friedrich Krause and his influence in the Hispanic World, Köln, Böhlau Verlag, 1998; Enrique M. Ureña, Krause, educador de la Humanidad. Una biografía, Madrid, Universidad Pontificia Comillas (UPCO), 1991; Klaus Kodalle, K.C.F. Krause (1781-1832). Studien zu seiner Philosophie und zum Krausismus, Hamburg, Felix Meiner Verlag, 1985; Juan López Morillas, El krausismo español, perfil de una aventura intelectual, Madrid, Fondo de Cultura Económica, 1980.

${ }^{4}$ Enrique. M. Ureña, «Prólogo», en: Antolín Sánchez Cuervo, El pensamiento krausista de Guillaume Tiberghien, Madrid, UPCO, 2004, p. 14.

${ }^{5}$ Antolín Sánchez Cuervo, Krausismo en México, op. cit., p. 14.
} 
por ser una filosofía práctica y reformista comprometida con el cambio. En 1897, al retirarse de la Universidad Libre, Tiberghien se refirió al krausismo como el «arsenal del liberalismo» ${ }^{6}$. Se trataba no sólo de comprender y de explicar al hombre y al mundo, sino de transformarlos. Así, los krausistas fueron, con frecuencia, educadores y políticos, "filósofos de acción», al decir de Pierre Jobit ${ }^{7}$. Lo mismo sucedió en el Río de la Plata ${ }^{8}$.

Por todo lo expuesto, se comprende que los krausistas volvieran una y otra vez al tema de la importancia práctica de la filosofía. Para vivir, para encarnar determinados ideales o valores había que conocerlos y reflexionar sobre ellos. ¿Cuáles eran estos ideales? Por un lado, la reforma de la sociedad en paz, para alcanzar la «Alianza de la Humanidad», como culminación de la historia; por otro lado, el estímulo de la sed de virtud moral, pues sólo si cada hombre cambiaba podría haber cambio social. Se trataba de alcanzar la "pura y completa humanidad», expresión utilizada por Krause, en 1811, en su obra más emblemática, Ideal de la Humanidad para la vida?. La vía era el cambio en "armonía», término de claras connotaciones masónicas y un concepto fundamental en el krausismo. El hombre era un "ser de armonía» y la historia un proceso hacia la «edad de la armonía». El principio «unir sin confundir, distinguir sin separar»-que Krause y sus discípulos aplicaron a todas las realidades- suponía todo un programa. Implicaba el valor de lo diferente y la importancia de la organización armónica de los elementos distintos, pero no por ello opuestos.

En cuanto a las relaciones del krausismo y la laicidad -en tanto imperio de la neutralidad del Estado en relación a los diversos cultos y en tanto separación de la pertenencia ciudadana y religiosa ${ }^{10}-$,

6 «Manifestation jubilaire en I'honneur de M. Guillaume Tiberghien, professeur de philosophie à I'Université Libre. 1846-1897, 5 décembre 1897», en: Bulletin de I'Union des Anciens Etudiants de I'Université de Bruxelles, Bruselas, t. 2, $1^{\circ}$ enero 1899, p. 26.

7 Pierre Jobit, Les éducateurs de l'Espagne contemporaine, t. I, Les Krausistes, Paris, De Boccard, 1936, p. 277.

${ }^{8}$ Sobre las razones de la adhesión al krausismo: Susana Monreal, Krausismo en el Uruguay. Algunos fundamentos del Estado tutor, Montevideo, Universidad Católica del Uruguay, 1993; Elías Díaz, La filosofía social del krausismo español, $2^{\circ}$ ed., Valencia, Fernando Torres, 1983; John Bartier, "L'Université Libre de Bruxelles au temps de Théodore Verhaegen», en: Laïcité et franc-maçonnerie. Etudes rassemblées et publiées par Guy Cambier, Bruxelles, Université Libre de Bruxelles, 1981, pp. 13-72; Pierre Jobit, Les éducateurs de l'Espagne contemporaine, op. cit.

9 Enrique M. Ureña, «Krause y su ideal masónico: hacia la educación de la Humanidad», en: Historia de la Educación, Revista Interuniversitaria, Salamanca, nº 4, 1985.

10 Jean Baubérot y Micheline Milot, Laïcités sans frontières, Paris, Éditions du Seuil, 2011, p. 21. 
éstos sostuvieron la independencia de las diversas esferas de la acción humana. La Humanidad era concebida como un gran organismo vivo, formado por la totalidad de los individuos, organizados en diferentes unidades sociales relacionadas en forma armónica. Las asociaciones humanas respondían a los diversos objetivos que el ser humano persigue: la Virtud, la Belleza, el Derecho, la Religión, la Ciencia, el Arte, la Educación. Las asociaciones o alianzas que correspondían a cada una de estas esferas culminarían en la "Alianza de la Humanidad». En el campo de la Educación, dada la «solidaridad de los fines humanos» y la unidad original de la sociedad «sobre toda determinación interior de sus miembros y esferas», debía asegurarse la libertad de enseñanza, entendida como libertad de centros y como libertad de cátedra, y quedaba asegurada la independencia de la Educación tanto del Estado, la esfera del Derecho, como de la Iglesia o las Iglesias, la esfera de la Religión ${ }^{11}$.

En esta línea de pensamiento, en octubre de 1876, Francisco Giner de los Ríos y otros compañeros liberales y krausistas fundaron en Madrid la Institución Libre de Enseñanza, y el 7 de marzo de 1877 apareció el primer número del Boletín de la Institución Libre de Enseñanza. La publicación incluía un encabezamiento sugerente: «La Institución Libre de Enseñanza es completamente ajena a todo espíritu e interés de comunión religiosa, escuela filosófica o partido político; proclamando tan solo el principio de la libertad de la ciencia, y de la consiguiente independencia de su indagación y exposición respecto de cualquiera otra autoridad que la de la propia conciencia del Profesor, único responsable de sus doctrinas» ${ }^{12}$.

En resumen, en el campo pedagógico, los krausistas representaron una tercera vía educativa, emancipada de la Iglesia y del Estado, que se inspiraba en el armonicismo fundamental de Krause, en su concepción metafísica panteísta y en su compromiso masónico. Esta tercera vía, señala Pedro Álvarez Lázaro, «en su línea básica de desarrollo no perseguía soluciones estatalistas ni confesionales al problema educativo, sino que, respetando las creencias individuales, pretendía una formación humana libre, universalista, tolerante y armónica» ${ }^{13}$.

\footnotetext{
${ }^{11}$ Nicolás Salmerón, «La libertad de enseñanza», en: Boletín-Revista de la Universidad de Madrid, Madrid, 1869, p. 58.

12 Boletín de la Institución Libre de Enseñanza (BILE), Madrid, 7 marzo 1877.

13 Pedro F. Álvarez Lázaro, "Krausistas, institucionistas y masones en la España del siglo XIX», en: Pedro F. Álvarez Lázaro y José Manuel Vázquez-Romero, Krause, Giner y la Institución Libre de Enseñanza. Nuevos estudios, Madrid, UPCO, 2005, p. 139.
} 
En relación con Fröbel, la coincidencia con Krause se dio en el planteo de una educación integral y en el desarrollo de «la pura y completa humanidad». Dados los proyectos que analizaremos más adelante, interesa la relación que existió entre Krause y Fröbel. Krause fue uno de los primeros en reconocer públicamente el valor de las ideas de Fröbel, lo que indujo al pedagogo a visitar al primero en Gotinga, en 1828, y a tomar del Ideal de la Humanidad las bases filosóficas para su sistema educativo. Por otra parte, Krause acercó a Fröbel a la obra de Comenio y a la educación de la primera infancia. Estas coincidencias tendrían su culminación en la estrecha colaboración que existió entre krausistas y fröbelianos a partir de $1869^{14}$. Esta afinidad y las relaciones existentes entre krausistas, institucionistas y pedagogía fröbeliana motivaron la utilización, por parte de algunos investigadores, del término «krausofröbelismo» ${ }^{15}$.

A partir de la década de 1870, círculos pedagógicos españoles y belgas, innovadores y de inspiración krausista, desarrollaron relaciones significativas con grupos de educadores rioplatenses. Dichas relaciones tuvieron efectos detectables en el desarrollo del concepto y de la praxis de una educación integral, en la renovación de los métodos pedagógicos y en los avances de la educación preescolar. En lo que sigue analizaremos tres casos: en primer lugar, la difusión de las obras de Guillermo Tiberghien, especialmente las que tratan temas educativos y éticos; luego, las relaciones desarrolladas por pedagogos rioplatenses con grupos de renovación pedagógica de Bélgica y España; finalmente, el desarrollo de las propuestas «krausofröbelianas» en las publicaciones oficiales de la enseñanza de Argentina y Uruguay.

\section{La difusión de las obras del belga Tiberghien}

Discípulo de Enrique Ahrens en la Universidad Libre de Bruselas, Guillermo Tiberghien (Bruselas, 1819 - SaintJosse-ten-Noode, 1901) adoptó la filosofía krausista y se mantuvo fiel a ella, con el fervor de un creyente. En poco tiempo, se transformó en el símbolo de la Universidad Libre y de su enseñanza; el rector fundador, Teodoro Verhaegen, lo llamó «el símbolo de la Universidad». Doctorado en Filosofía en 1843, inició inmediatamente su carrera docente y, cuando Ahrens dejó Bruselas en 1848, Tiberghien lo sucedió en la cátedra ${ }^{16}$. «La filosofía de Krause huele a ideal. Ella goza, en la región de las

\footnotetext{
${ }^{14}$ Pedro F. Álvarez Lázaro, «Krausistas, institucionistas y masones...», op. cit., p. 140.

${ }^{15}$ Enrique M. Ureña, El krausismo alemán. Los congresos de filósofos y el krausofröbelismo (18331881), Madrid, UPCO, 2002, pp. 155-321.

${ }^{16}$ Susana Monreal, Krausismo en el Uruguay..., p. 50-56.
} 
ideas eternas, de lo bueno, de lo bello, Popular (BSAEP), del Club Católico de de lo verdadero, de lo justo, ella aborda Montevideo (BCCM), del Ateneo de sin temores la metafísica», afirmó en su Montevideo (BAM), de la Compañía de última clase en la Universidad Libre ${ }^{17}$. Jesús (BSJ), de la Universidad Católica El compromiso de Tiberghien se extendió del Uruguay (BUCU). En Buenos Aires a otras áreas de acción. Fue militante del se relevaron ocho bibliotecas: Biblioteca partido Liberal, miembro fundador de la Libre Pensée, sociedad de librepensadores fundada en 1863, e integrante de la Ligue de l'Enseignement, creada en 1864. En la defensa del programa liberal puso un especial acento en los principios de obligatoriedad y laicidad de la enseñanza primaria, a los que dedicó numerosos estudios en su calidad de docente, de político y de masón, siendo miembro de la logia de «Los Amigos Filántropos».

Varias obras del profesor belga, sobre temas educativos y morales, fueron conocidas y leídas tanto en Montevideo como en Buenos Aires. En Montevideo, se realizó el relevamiento de las obras de Tiberghien en once bibliotecas: Biblioteca Nacional (BNU), Central de Enseñanza Secundaria (BCES), Pedagógica Central-Museo Pedagógico (BPC), del Poder Legislativo (BPL), de la Facultad de Derecho y Ciencias Sociales de la Universidad de la República (BFD), de la Facultad de Humanidades y Ciencias de la Educación. Universidad de la República (BFHUCE), de la Sociedad de Amigos de la Educación Nacional (BNA), Biblioteca Nacional de Maestros (BNM), del Colegio Nacional (BCN), del Museo Mitre (BMM), Biblioteca Tornquist del Banco Central de la República Argentina (BT-BCRA), de la Facultad de Derecho de la Universidad de Buenos Aires (BFD-UBA), de la Facultad de Filosofía y Letras de la Universidad de Buenos Aires (BFILO-UBA) y de la Universidad Católica Argentina (BUCA). En Montevideo se detectaron doce títulos de Tiberghien y veintisiete ejemplares. En Buenos Aires, se encontraron quince títulos y veintiséis ejemplares. Presentamos los resultados obtenidos en relación a seis obras particularmente significativas:

Ensayo teórico e histórico sobre la generación de los conocimientos humanos en sus relaciones con la moral, la política y la religión. Traducción de Alejo García Moreno. Madrid, Francisco Góngora Editor, [1875].

- Buenos Aires - BNA / BNM (2) - Montevideo - BNU / BFD / BCES / $\mathrm{BPC} / \mathrm{BFUHCE} / \mathrm{BSJ} / \mathrm{BAM}(7)$

\footnotetext{
17 «Manifestation jubilaire en I'honneur de M. Guillaume Tiberghien...», op. cit. p. 25-26.
} 
Introduction à la philosophie et préparation à la métaphysique. Etude analytique sur les objets fondamentaux de la science; critique du positivisme. Bruxelles et Liège, Auguste Decq, 1868.

- Buenos Aires - BNA / BFILO-UBA (2)
Elementos de ética o filosofía moral arreglados para la $2^{a}$ enseñanza por Hermenegildo Giner. Madrid, Librería de Alfonso Durán, 1873.

- Buenos Aires - BNA / BNM / BFD-UBA (3) - Montevideo - BNU / BCES / BFUHCE (3)
Introducción a la filosofia y preparación a la metafisica. Estudio analitico sobre los objetos fundamentales de la ciencia critica del positivismo. Traducción española de Vicente Piñó y Vilanova, Madrid, Imprenta de la Revista de Legislación, 1875.

- Buenos Aires - BNA (2)

- Montevideo - BNU / BCES / BPL (3)

Enseignement et philosophie. Bruxelles, G. Mayolez, 1873.

- Buenos Aires - BFILO-UBA (1)

La enseñanza obligatoria. Traducción y notas biográficas del autor por Hermenegildo Giner de los Ríos. Madrid, Anlló y Rodríguez, 1874. Iincluye los estudios sobre instrucción obligatoria, publicados en Enseignement et philosophie.

- Buenos Aires - BNA / BNM / BCN / BFILO-UBA (4)

- Montevideo - BFD / BAM (2)

Estudios sobre filosofía. Traducción de Alejo García Moreno. Madrid, Imprenta M. Minuesa, 1875. Reúne los artículos de tema filosófico de Enseignement et philosophie.

- Buenos Aires - BUCA (1)
Los mandamientos de la humanidad o la vida moral en forma de catecismo según Krause, Madrid, Imprenta de Manuel Minuesa, 1875.

- Buenos Aires - BNA / BFILO-UBA (2)

- Montevideo - BNU / BCES / BAM (3)

Éléments de morale universelle à l'usage des écoles lä̈ques. Bruxelles, Gustave Mayolez 1879.

- Montevideo - BCES (1)

Moral elemental para uso de las escuelas. Traducción por Hermenegildo Giner de los Ríos, Madrid, Imprenta de la Gaceta Universal, 1880.

- Buenos Aires - BFILO-UBA (1)

Los títulos de Tiberghien que circularon en las capitales del Río de la Plata coinciden claramente. Además, las dos primeras obras citadas, el Ensayo teórico e histórico, de 1844, y la Introducción a la filosofía, de 1868 , son las que el autor consagró a la exposición del sistema krausista. Traducidas al español, ambas alcanzaron una difusión destacable en América Latina y en el Río de la Plata. Debe también mencionarse que las demás obras citadas tratan temas de 
educación y de moral, y se vinculan con la promoción de la educación laica.

De las seis obras citadas cinco fueron traducidas al español, entre 1869 y 1875. Se trata de la etapa del «krausismo triunfante» en España, de consolidación del proyecto institucionista, de fuerte influencia en la Universidad de Madrid $y$ con gran presencia en las ediciones ${ }^{18}$. Se tradujeron entonces nueve obras de Tiberghien, que se difundieron ampliamente en el mundo cultural hispano y que, en el Río de la Plata, realimentaron la definición krausista de profesores y políticos. En Montevideo, Prudencio Vázquez y Vega tomó el Ensayo teórico e histórico como guía para la elaboración del programa de los cursos de Filosofía que dictó en el Ateneo ${ }^{19}$. En Buenos Aires, la Introducción a la filosofía fue libro de cabecera de Hipólito Yrigoyen ${ }^{20}$.

\section{Las relaciones con los grupos belgas y españoles de renovación pedagógica}

En el último cuarto del siglo XIX, las relaciones culturales entre España e Hispanoamérica adquirieron un nuevo impulso. La celebración del cuarto centenario del descubrimiento de América y los preparativos previos motivaron el retorno afectivo de los latinoamericanosiberoamericanos a sus orígenes, de los que habían renegado por largo tiempo. Esta nueva tendencia intensificó las relaciones con los grupos de educadores españoles; por otra parte, los maestros habrían sido los inmigrantes espańoles culturalmente más calificados que llegaron en mayor número a América y al Cono Sur ${ }^{21}$.

A comienzos de 1889, llegó a Buenos Aires Antonio Atienza y Medrano ${ }^{22}$ (Almería, 1852 - Buenos Aires, 1906), aprovechando las facilidades concedidas por el gobierno

${ }^{18}$ Susana Monreal, Krausismo en el Uruguay, op. cit., pp. 108-110.

19 ĺdem, p. 58.

${ }^{20}$ Arturo A. Roig, Los krausistas argentinos, op. cit., p. 66.

${ }^{21}$ Gabriela Ossenbach Sauter, «Pedro Alcántara García y las relaciones pedagógicas entre España e Hispanoamérica a finales del siglo XIX», en: Historia de la Educación. Revista Interuniversitaria, Salamanca, 11, enero-diciembre 1992.

22 Sobre Antonio Atienza y Medrano, ver: Ignacio García, Emigración y patriotismo. La colonia española en Buenos Aires ante la crisis de 1898, Tesis de Doctorado inédita, Departamento de Español y Estudios Latinoamericanos, University of New South Wales, Sidney, Australia, 1997, pp. 214-276 y 364-385; Marcela García Sebastiani, "Antonio Atienza y Medrano, institucionista en otras tierras», en: M. García Sebastiani (ed.), Patriotas entre naciones: Elites inmigrantes españolas en Argentina, Madrid, Editorial Complutense, 2010. 
argentino. Era licenciado en Derecho y krausista de neta filiación institucionista. Discípulo de Nicolás Salmerón, había acompañado a Francisco Giner de los Ríos en la fundación de la Institución Libre. Con Urbano González Serrano, Augusto Gómez de Linares, Alfredo Calderón, Hermenegildo Giner y otros perteneció a la tercera generación del krausismo, la que convivió con el positivismo, admitiendo el valor de sus avances científicos pero enfrentando sus ribetes materialistas.

Según Ignacio García, Atienza podría haber llegado especialmente contratado para promover el institucionismo en Argentina. Así explica su inmediata designación como docente en el Colegio Nacional y su rápido ingreso en El Monitor de la Educación Común, como colaborador primero y como redactor más tarde ${ }^{23}$. Su rápida inserción en los círculos pedagógicos también podría explicarse por su condición de masón. En efecto, Atienza se había iniciado en la logia «El Porvenir» de Madrid ${ }^{24}$.

A lo largo de casi seis años, entre julio de 1889 y abril de 1895, Atienza se erigió en portavoz de propuestas innovadoras relacionadas con el desarrollo del método activo y con el nuevo rol del maestro. En este lapso, escribió 31 artículos; 22 de ellos, artículos editoriales. Trató temas diversos, muy relacionados con las propuestas renovadoras de la Institución Libre: colonias escolares de vacaciones, excursiones pedagógicas, higiene escolar, educación física, educación estética, educación de los obreros.

Durante el mismo período, en las capitales del Plata, se publicaron con cierta frecuencia artículos de educadores vinculados a la Institución Libre y textos provenientes del Boletín de la Institución Libre de Enseñanza (BILE). El primer artículo procedente del Boletín, publicado en El Monitor, fue un texto de Francisco Giner de los Ríos, en $1885^{25}$. Si bien no es fácil precisar la procedencia de los textos publicados en El Monitor, dado que no siempre aparece este dato, en octubre de 1891 se detecta uno nuevo tomado del $B I L E$. Se trata de «El arte de hacer hablar y pensar a los nińos», de Pedro de Alcántara García, publicado en Montevideo en diciembre de $1890^{26}$. En cuanto a las revis-

\footnotetext{
${ }^{23}$ Ignacio García, Emigración y patriotismo, op. cit., p. 370; El Monitor de la Educación Común (en adelante El Monitor), Buenos Aires, 31/07/1889, p. 1; El Monitor, 30/04/1895, p. 1.

24 Pedro F. Álvarez Lázaro, «Krausistas, institucionistas y masones», op. cit., p. 168.

25 «Francisco Giner. Un peligro de toda enseñanza», El Monitor, septiembre de 1885, pp. 870-872. Se aclara: «Extracto que aparece en el Boletín de la Institución libre de Enseñanza de Madrid, de un libro en preparación, sobre el Método activo».

${ }^{26}$ Pedro de Alcántara García, «El arte de hacer hablar y pensar a los niños», El Monitor, octubre de 1891, pp. 1117-1121.
} 
tas educativas de Montevideo, el Boletín de Educación Primaria publicó desde su fundación en 1889 numerosos artículos del BILE. Los primeros fueron «Las colonias escolares», de Manuel B. Cossio; «Desarrollo de las ideas pedagógicas en Francia desde 1870", de Henri Marion, de la Universidad de París; y «El arte de hacer hablar y pensar a los niños», de Pedro de Alcántara García ${ }^{27}$. Los artículos del BILE aparecieron hasta 1898 , si bien desde 1891 el material proveniente de La Escuela Moderna fue netamente predominante.

Durante este período se publicaron también artículos provenientes de los círculos educativos belgas, que habían inspirado los proyectos españoles. En 1864 se había fundado en Bruselas la Ligue de
l'Enseignement, promotora de la Escuela Modelo "Charles Buls», dirigida desde 1878 por Alexis Sluys ${ }^{28}$. Sus integrantes eran liberales belgas, muchos de ellos graduados en la Universidad Libre de Bruselas, y masones, relacionados también con la Libre Pensée. Estos grupos innovadores apoyaron la edición de la Revue Pédagogique Belge, que circuló también en el Río de la Plata. Esta revista se editó, entre enero de 1888 y diciembre de 1895, bajo la dirección de Auguste Smets ${ }^{29}$. Pretendía responder a las inquietudes no sólo de los educadores sino también del público profano interesado en temas educativos.

En Montevideo, la Revue Pédagogique Belge se recibía en canje por el Boletín de Enseñanza Primaria $a^{30}$, en el que se

\footnotetext{
${ }^{27}$ Manuel B. Cossio, «Las colonias escolares», Boletín de Enseñanza Primaria, Montevideo, octubre de 1889, pp. 314-325 y noviembre de 1889, pp. 342-347; H. M. Marion, «Desarrollo de las ideas pedagógicas en Francia desde 1870", BEP, noviembre de 1890, pp. 281-294; Pedro de Alcántara García, «El arte de hacer hablar y pensar a los niños», BEP, diciembre de 1890, pp. 341-351.

28 Alexis Sluys (1849-1936): maestro formado en Bruselas que, en 1878, sucedió a Carlos Buls como director de la Escuela Modelo. A partir de 1881, durante 30 años, dirigió la nueva sección de maestros de Bruselas, instalada en la Escuela Modelo. En 1872 fue iniciado en la logia de «Los Amigos Filántropos». Biographie Nationale, Bruxelles, Emile Bruylant, 1958, 30, col. 765-767; Pierre Defosse (dir.), Dictionnaire historique de la laïcité en Belgique, Bruxelles, Editions Luc Pire-Fondation Rationaliste, 2005, p. 254.

${ }^{29}$ Auguste Smets (1850-1936): educador y político liberal belga, que enseñó en la Escuela Modelo y en la sección normal de maestros. En 1875 fundó y dirigió L'Avenir y, entre 1888 y 1895, la Revue Pédagogique Belge. Presidió la Ligue de l'Enseignement e integró la logia «Verdaderos Amigos de la Unión y del Progreso reunidos». Pierre Defosse (dir.), Dictionnaire historique de la laïcité, op. cit., p. 255. 30 "Nómina de los periódicos recibidos en canje con el Boletín de Enseñanza Primaria», BEP, julio 1892, p. 37-41.
} 
publicaron artículos de Alexis Sluys y de Auguste Smets ${ }^{31}$. Por otra parte, eran frecuentes las noticias provenientes de la Escuela Modelo y de la Escuela Normal de Bruselas. En Buenos Aires, si bien no pudimos acceder a las listas de canje de $E l$ Monitor, detectamos aportes de la Revue Pédagogique Belge. En 1890 fue publicado el artículo "Un Jardín de Infantes» de Félix Ley ${ }^{32}$ y en 1893 el artículo «Lo que debe ser la escuela» de Alexis Sluys. Los contenidos pedagógicos son valiosos, pero interesan sobre todo los comentarios relacionados con la ley liberal y laica de educación -ley Van Humbeeck-, de 1879, y la posterior «lucha escolar» en Bélgica. En 1893, Sluys sostenía:

«La escuela primaria pública debe ser en cada población y en cada distrito de la ciudad la CASA DEL PUEBLO; esta es su misión social; cuando la cumpla, hará dar a la civilización, al progreso pasos agigantados. Y el maestro no será más, desde entonces, el emisario de las lamentables represalias de nuestra miserable política, de miras estrechas y de intereses egoístas; no será más discutido, calumniado, vilipendiado por los reaccionarios (...) pues cumpliendo en la sociedad la misión civilizadora que acabamos de bosquejar, la escuela y el maestro serán enérgicamente sostenidos por el pueblo, que sabrá apreciarlos y defenderlos contra los retrocesos de la reacción» ${ }^{33}$.

\section{Las propuestas fröbelianas en las publicaciones oficiales}

El método de educación de Federico Fröbel, a través del Kindergarten o jardín de infancia, se difundió por Europa en la segunda mitad del siglo XIX. La propuesta se basaba en la actividad personal y el juego, y tendía al desarrollo integral de la personalidad de cada niño. Inspirados en Rousseau y en Pestalozzi, Fröbel y sus seguidores sostenían una concepción

${ }^{31}$ Alexis Sluys, "La enseñanza de los trabajos manuales en las escuelas primarias de niños», BEP, marzo 1890, pp. 194-207; mayo de 1890, pp. 307-327 y agosto de 1890, pp. 86-91; A. Sluys, «El arte en la escuela primaria», BEP, diciembre de 1892, pp. 298-324; «Comentario sobre artículo de Alexis Sluys, La enseñanza de los trabajos manuales en Suecia y en Bélgica», BEP, febrero de 1893, p. 143; «Comentario sobre artículo de Auguste Smets, El ahorro escolar», BEP, noviembre de 1891, p. 240. 32 Félix Ley (1846-1919): maestro diplomado en la Escuela Normal de Huy, trabajó en la Escuela Modelo y en la Escuela Normal de Bruselas. Era masón, integró la Ligue de l'Enseignement y la Libre Pensée, y colaboró en las revistas Progrès y L'Avenir, antes de fundar L'enseignement pratique. Pierre Defosse (dir.), Dictionnaire historique de la laïcité, op. cit, p. 187.

${ }^{33}$ Alexis Sluys, "Lo que debe ser la escuela», El Monitor, 15 junio 1893, p. 147. Las mayúsculas son del autor. 
romántica del niño, considerado un ser bueno por naturaleza y moralmente superior al adulto. La nueva metodología se vinculaba con la concepción del nuevo "ciudadano", liberado de las relaciones tradicionales de autoridad y de respeto, que se manifestaría plenamente en las revoluciones europeas de $1848^{34}$.

Esta propuesta innovadora llegó a las capitales del Río de la Plata algunas décadas más tarde, por vía hispánica. Sin desconocer antecedentes que se remontan a la década de 1870, ni la obra de Sara Chamberlain de Eccleston, la gestión de Antonio Atienza como redactor de El Monitor coincidió con la publicación de artículos de dos pedagogos europeos, de perfil diverso y de diferente nacionalidad, que tenían en común su relación con los krausistas: la baronesa Bertha von Marenholtz-Bülow y el profesor Pedro de Alcántara García. La publicación, en 1873, en Sevilla, de la traducción de la obra El niño y su naturaleza de la baro- nesa Marenholtz-Bülow precedió en un año a la divulgación del libro de Pedro de Alcántara García, Fröbel y los Jardines de Infancia, el primero que el educador español consagrara al tema ${ }^{35}$.

Bertha von Marenholtz-Bülow (Brunswick, 1816 - Dresde, 1893), dedicada propagandista del método de Fröbel, participó en los dos Congresos de filósofos krausistas de Praga y Frankfurt, y fue uno de los pilares de la Asociación General para la Educación, fundada en Dresde, en 1871, presidida por Hermann von Leonhardi, discípulo directo y yerno de $\mathrm{Krause}^{36}$. Entre el 31 de marzo de 1892 y el 31 de diciembre de 1893, fueron publicados en El Monitor, seis largos artículos de Marenholtz-Bülow: «Idea fundamental del sistema de Fröebel» (sic), 31/03/1892, pp. 185-189; «Sistema de Fröebel. II. Ley de Fröebel. Mediación de opuestos», 31/05/1892, pp. 255-258; «Sistema de Fröebel. III. Relaciones de la Ley de Equilibrio», 30/06/1892, pp.

\footnotetext{
${ }^{34}$ Gunilla-Friederike Budde, «Histoire des jardins d'enfants en Allemagne», en: Jean-Noël Luc (dir.), L'école maternelle en Europe: XIXe-XXe siècles, Paris, Institut National de Recherche Pédagogique, 1999. Disponible en: http://ife.ens-lyon.fr/publications/edition-electronique/histoire-education/RH082. pdf (último ingreso: 12/07/2014).

${ }^{35}$ Carmen Colmenar Orzaes, «Les écoles maternelles en Espagne au XIXe siècle», en: Jean-Noël Luc (dir.), L'école maternelle en Europe, op. cit., p. 134; Carmen Colmenar, «La introducción de los jardines de infancia en España. Aportaciones de Pedro de Alcántara y Eugenio Bartolomé y Mingo», en: Ma del Carmen Sanchidrián Blanco y J. Ruiz Berrio (coord.), Historia y perspectiva actual de la educación infantil, Barcelona, Editorial Graó, 2010, p. 138.

${ }^{36}$ Enrique M. Ureña, El krausismo alemán. Los congresos de filósofos y el krausofröbelismo (18331881), Madrid, UPCO, 2002, pp. 120-138 y 280-316.
} 
276-278; «Método de Fröebel. IV. La ley de Fröebel como ley de la educación", 31/10/1892, pp. 407-410; «Sistema de Fröebel. V. El niño como perteneciendo a Dios, a la naturaleza y a la Humanidad. VI. El Kindergarten. VII. Vista general del Kindergarten", 31/03/1893, pp. 53-55; «Sistema de Fröebel. VIII. Consideraciones sobre los dones y ocupaciones. IX. El tercer Don. X. El cuarto Don. XI. El quinto y sexto Don", 31/12/1893, pp. 492-494.

Por su parte, Pedro de Alcántara García Navarro (Córdoba, 1842 - Madrid, 1906) fue también destacado promotor del fröbelismo en Espańa y en Hispanoamérica. Fue profesor de Pedagogía especial de párvulos por el sistema Fröbel, tanto en las Escuelas Normales Centrales de Maestros y Maestras, como en la Escuela de Institutrices de Madrid ${ }^{37}$. En 1879 publicó el Manual teórico-práctico de educación de párvulos según el método de los jardines de la infancia de F. Fröbel, al que siguieron otras obras sobre el tema. Fue además colaborador asiduo del BILE, si bien, en 1891, fundó su propia revista, $L a$ Escuela Moderna. Esta publicación, que García Navarro dirigió hasta su muerte, llevaba el subtítulo de Revista Pedagógica
Hispano-Americana, lo que resulta revelador $^{38}$. Los artículos de García aparecieron en El Monitor a partir de abril de 1891, siendo Atienza redactor de la publicación: «El arte de hacer hablar y pensar a los niños», 30/04/1891, pp. 1117-1121; «De la enseñanza cíclica», 31/07/1893, pp. 217-220; «La interrogación socrática», 31/03/1894, pp. 782-786, «El arte de preguntar», 31/07/1894, pp. 969-972; «El arte de hacer hablar y pensar a los niños», 31/07/1895, pp. 145-150; «España: Revista de Enseñanza y Cuestiones de Pedagogía», 31/10/1896, pp. 599-605; «La enseñanza musical en las escuelas», 31/01/1903, pp. 1100-1104.

En Montevideo, detectamos una situación muy similar en algunos aspectos y diferente en cuanto a las modalidades de la influencia recibida. El fröbelismo tuvo también especial difusión a través de la baronesa Marenholz Bülow y de Pedro de Alcántara García. La pedagoga alemana apareció con cierta frecuencia en el Boletín de Educación Primaria, si bien no se publicaron textos de su autoría. José H. [Henriques] Figueira, primer director del Boletin y su constante promotor, manifestó especial adhesión a las propuestas y a la figura de la baronesa. A las primeras

\footnotetext{
37 Juan Félix Rodríguez Pérez, «Un maestro de maestros. Pedro de Alcántara García Navarro (18421906) y la Sociedad Protectora de los Niños de Madrid». Disponible en: http://www.forodeeducacion. com/numero9/010.pdf (último ingreso: 24/08/2011).

38 Gabriela Ossenbach Sauter, «Pedro Alcántara García y las relaciones pedagógicas entre España e Hispanoamérica», op. cit., pp. 135-136.
} 
noticias sobre el desarrollo de los Jardines de infantes en Europa, publicadas en 1889, siguió el informe sobre la visita de Figueira a la «señora Marenholz-Bülow» -y también a Alexis Sluys- con motivo de su viaje a Europa en 1891. La admiración del educador uruguayo se confirmó en 1893, cuando, con motivo de la muerte de Marenholz-Bülow, publicó una extensa y laudatoria nota: «Tuve la honra de conocer a esta venerable señora (...) y me fue muy grato visitar los Jardines de Infancia de Dresde, que reputo como los mejores de Alemania ${ }^{39}$.

En cuanto a García Navarro, sus artículos fueron publicados desde la década de 1870. En 1878, El Eco del Maestro publicó en varias entregas su obra Froebel $y$ los jardines de la infancia ${ }^{40}$. García fue además el autor extranjero con más artículos publicados en el Boletín, algunos provenientes del BILE y otros -la mayoría- de La Escuela Moderna: «Educación, instrucción enseñanza y cultura», agosto 1890, pp. 92-97; «El arte de hacer hablar y pensar a los niños», diciembre 1890, pp. 341-351; «El arte de preguntar», octubre 1892, pp. 189-196; "La interro- gación socrática como instrumento del método activo", abril 1893, pp. 238-247; «Necrología: Don Mariano Carderera y Potó», mayo 1893, pp. 297-303; «La enseñanza de la pedagogía en las escuelas normales», diciembre 1893, pp. 451-465; "Los exámenes en las escuelas primarias", agosto 1895, pp. 95-102.

Por otra parte, las obras de Pedro de Alcántara García se hallan en buen número en la Biblioteca Nacional Argentina y en la Biblioteca Nacional de Maestros, en Buenos Aires, y en la Biblioteca del Museo Pedagógico de Montevideo, tradicionalmente vinculada a la primera Escuela Normal. Este hecho evidencia la utilización de los textos de García como manuales en las Escuelas Normales rioplatenses y prueban su peso en la divulgación de la obra de Fröbel en el Cono Sur. En la Biblioteca Nacional Argentina se encuentran trece ejemplares de obras de García Navarro, en la Biblioteca Nacional de Maestros, once ejemplares y otros títulos. Doce títulos se hallan en Montevideo, siete coinciden con los ubicados en Buenos Aires. El Manual teórico-práctico de educación de párvulos según el método

\footnotetext{
39 "Jardines de infantes», $B E P$, agosto de 1889, p. 139; «José H. Figueira en Europa», BEP, mayo de 1891, p. 434; José H. Figueira, «Necrología: La baronesa de Marenholz-Bülow», BEP, abril de 1893, pp. 257-259.

40 Pedro de Alcántara García, «Froebel y los jardines de la infancia», El Eco del Maestro, Montevideo, 01/09/1878, pp. 38-39, 15/09/1878, pp. 53-55, 29/09/1878, pp. 68-69; 15/10/1878, pp. 77-78; 15/12/1878, pp. 108-109; y 30/12/1878, p. 114.

${ }^{41}$ En dos ediciones: Madrid, Colegio Nacional de Sordomudos y de Ciegos, 1879 y Madrid, Hernando, 1883.
} 
de los jardines de infancia de F. Fröbel ${ }^{41}$ se encuentra en todas esas bibliotecas.

La particularidad uruguaya, en relación con la difusión del fröbelismo, fue el viaje realizado en 1890 por Enriqueta Compte y Riqué, para estudiar la organización de los Kindergärten en Francia, Bélgica, Alemania y Suiza. Nacida en Barcelona en 1866, Compte y Riqué había llegado a Uruguay en su primera infancia. En 1884 obtuvo el diploma de maestra e inició una carrera destacada. A partir de su viaje a Europa, se organizaron los «cursos de Jardines de Infancia» para formar maestras, en octubre de 1890, y se instaló el primer Jardín Fröbel, en 1892. A lo largo del viaje, Compte y Riqué escribió memorias, publicadas por el Boletín de Montevideo y por El Monitor de Buenos Aires ${ }^{42}$.

\section{A modo de cierre}

Aun cuando el valor adjudicado a la educación en la promoción del desarrollo social no fue una propuesta exclusiva del krausismo, debe subrayarse la energía que los krausistas europeos e iberoamericanos invirtieron en la tarea educativa y las profundas esperanzas que depositaron en ella. El desarrollo de la educación debía conducir a la realización personal plena y debía crear las bases para una participación democrática real. En tal sentido, la educación para la libertad y para el amor, libre de los controles del Estado y de la Iglesia, era uno de los medios privilegiados para alcanzar la «edad de la armonía». La educación, junto con los avances científicos y la acción política, eran las vías seguras del desarrollo de cada persona y de la comunidad. En tal sentido, en el campo educativo, el espíritu innovador y la búsqueda de autonomía de la esfera de la Educación fueron ideas distintivas de los krausistas. Por esta razón fueron impulsores de la educación laica, con un talante respetuoso y tolerante en España, con un carácter marcadamente anticlerical y beligerante en Bélgica, con matices propios en las naciones hispanoamericanas.

Por otra parte, a partir del mismo Krause, el sistema incluyó una pedagogía

\footnotetext{
42 «Memoria correspondiente al mes de diciembre de 1889 por la Srta. Enriqueta Compte y Riqué, comisionada por la Dirección General de Instrucción Primaria para estudiar los Jardines de Infantes», BEP, febrero de 1890, pp. 113-144; “Memoria... enero de 1890...», BEP, marzo de 1890, pp. 169193; «Memoria... febrero de 1890...», BEP, abril de 1890, pp. 235-257; "Memoria... marzo de $1890 \ldots$... BEP, junio de 1890, pp. 413-430; «Memoria... abril de 1890...», BEP, julio de 1890, pp. 8-21; «Memoria... mayo de 1890...», BEP, agosto de 1890, pp. 72-84; «Memoria... junio de 1890...», BEP, septiembre de 1890, pp. 129-141; «Correo del Exterior. Francia. Jardines de Infantes», El Monitor, 30/04/1899, pp. 1324-1337; «Correo del Exterior. Bélgica. Jardines de Infantes», El Monitor, 15/05/1890, pp. 1383-1394.
} 
completa e innovadora. Krause distinguió educación de instrucción, y diferenció la educación de los niños y la formación mutua entre iguales. Propuso la educación para la autoeducación, la educación fundada en la libertad y en el amor, la coeducación de los sexos. Destacó el valor de la educación religiosa, el rol de la familia en la educación y la importancia de la educación de la mujer y del varón en condiciones iguales. Por último, insistió en el juego como vía privilegiada de educación del niño desde su nacimiento; he aquí su coincidencia con Fröbel.
Todos estos rasgos del krausismo se manifestaron en los ambientes educativos de Buenos Aires y Montevideo, y respaldaron, con matices diversos, los cambios pedagógicos del período entre siglos. Asimismo, esta apertura pedagógica coincidió con los avances secularizadores en el campo de la educación. En el Río de la Plata, con suceso variado, las ideas y las propuestas europeas de inspiración krausista fueron una fuente de inspiración del proceso educativo de modernización y de secularización, entendido como emancipación de la fundamentación moral de las tradiciones religiosas. 